\title{
The Reconstruction of Translator's Identity under the Notion of "Fusion of Horizons"
}

\author{
Chang-hong Zhai \\ School of Foreign Languages Wuhan Polytechnic University, Wuhan, Hubei Province, China \\ 594561792@qq.com
}

\begin{abstract}
Traditional translation studies were based on three fundamental theories, namely, the principle of "faithfulness", the authority of the author and the binary opposition relations between the author and the translator as well as between the original text and the translated version. Therefore, the identity of the translator was defined as the servant, that is, "a servant of two masters", and as the "invisible man". In the 1980s, the emergence of the "cultural turn" opened up a new perspective and a broader horizon for translation studies. Based on the retrospect of the translator's identity in traditional translation theories, this paper is designed to analyze the reconstruction of the translator's identity under the notion of "fusion of horizons" in Gadamer's philosophical hermeneutics.

Index Terms - the translator's identity, the reconstruction, fusion of horizons
\end{abstract}

\section{Introduction}

Translation is an activity with a long history of several thousand years, whose contribution is absolutely obvious. However, the status of translators has not got the deserved attraction. There are indeed scholars dealing with the translators' status, but no systematical theory has been achieved. The history of the translation studies shows that the question of the translator is an eternal topic in translation. But because of the historical limitation, the width and depth of discussion about the translator are inadequate. Moreover, the identity of the translator has been reduced to a lower position because the emphasis is only put on the translated text and the comparison between the original text and its translated text. And in the field of translation study both at home and abroad, there have not existed quite systematic and adequate researches into the translator's identity under the notions of "fusion of horizons" and "horizon of expectations". Thus it is quite necessary to reconstruct the translator's identity by employing the notion of "fusion of horizons".

\section{The Translator's Position in the Traditional Translation Theories}

In the traditional translation studies, the translator's position had nearly been reduced to ignorance, whose identity had been regarded as "a servant serving two masters". The author had the absolutely full authority, while the translator was merely his loyal "translation machine". Moreover, the early reception theory and the reader response criticism had strongly emphasized the role of the reader, holding that it was the reader who decided the meaning of the text and that the translator was inevitably doomed to serve the reader. As a matter of fact, what mentioned above was not in accordance with the translators' actual subjectivity. Being the reader, the translator was supposed to mobilize his own literary abilities such as aesthetic judgment, emotion, imagination, etc. to interpret the blank points in the original-language texts; being the interpreter, he had the needs to exert his abilities of literary appreciation and literary criticism, to explore the ideological connotation and the aesthetic implication of the works, and to analyse the literary values and the social significance of the works. Under the traditional cultural notions, authorcenteredness and text-centeredness lay in authoritative positions. Either translation theories or translation criticism are abided by the principle of absolute faithfulness to the author and the original text. Researchers saw translation as a low- status occupation. Thus, they neglected the active role played by the translator, whose roles were considered as of importance.

In the traditional translation theories, people's understanding of translation had merely been restrained to a level of language conversion in a long period. The translator had generally been regarded as playing the role of "maiden", whose master was firstly the author or the original text as the incarnation of the author, and then the listener or the reader. The translator had been supposed not only to "convey" the actual meaning of what the author said and written, but also to make the listener and the reader understand and accept. The almost Utopian stickiness and pursuit of the concept of "faithfulness" had caused the translator to permanently live in the shadow of the author's authority.

Just like the relationship between the man and the woman in the history, the relation of the author and the translator also had to obey a kind of hierarchal rank, which honoured the upper and dishonoured the lower. "The translator and the woman had respectively put on the image of the weak in their own class: the translator was the author's maid; the woman is inferior to the man. [1]"... The author, like the man, represents the origin, primacy and creativity, holding the dominant position in the hierarchal order; whereas the translator, like the woman, is derivational, secondary, mechanical and uncreative, only being able to slavishly be obedient to the author. Therefore, Nicoie Ward Jouve [2] asserted that the translator had taken the position of the woman from the perspective of culture. The translator's living situation when he had been oppressed and overlooked in the hierarchal system had lasted for over one thousand years. The translator's voice had been delayed and deferred that though some different voices could sometimes be heard, their speech had always been marginalized in the context of the traditional translation discourse. 
In conclusion, in the traditional translation theory, "the translator was confined in the original-language meaning, grammar, structure, style and the original-language culture." [3], which led to the inevitable revealing of the translator's humble status. However, the translator's inferior status had been greatly improved under the notions of the Philosophical hermeneutics with Gadamer acting as its main representative and the reception theory with Jauss acting as its main representative. This paper will start from the notion of "fusion of horizons" to analyse the reconstruction of the identity of the translator.

\section{The Reconstruction of the Translator's Identity under the Notion of "Fusion of Horizons"}

\section{A. The Introduction about "Fusion of Horizons"}

Germany philosopher Gadamer was one of the representatives of the western post-modern hermeneutics. "Fusion of horizons" was one important notion in Gadamer's philosophical hermeneutics, which referred to the fusion of the author's and the reader's horizons. Hermeneutics, a new philosophy and cultural ideological trend prevailing in the west after the 1960s, was a kind of theory that explored meaning understanding and interpretation. Based on inheriting Heidegger's thoughts, Gadamer had deepened his understanding of interpretation and further developed Ontological hermeneutics into philosophical hermeneutics, thus fostering hermeneutics to flourish.

Gadamer [4] had put forward three philosophical principles in his famous work Wahrheit und Methode, namely, "historical interpretation", "fusion of horizons" and "effected history". And as a matter of fact, Gadamer's translation thoughts had been mainly embodied in those three philosophical principles mentioned above. The notion of "fusion of horizons" would primarily be employed in analyzing the reconstruction of the translator's identity in this paper.

\section{B. The Reconstruction of the Translator's Identity under the} Notion of "Fusion of Horizons"

Gadamer argued that any interpretation was conducted within the history and that either the subject of the interpretation or the object of the interpretation was a certain historical existence. The interpreter's understanding of the texts would never avoid the limit of the history. History was the essential fact that proved man's existence. He also held that the real understanding and interpretation was not to overcome its historical limitations, but to correctly evaluate and adapt to them. In Gadamer's opinion, interpretation was not a process of duplication and the real historical truth was of no great significance on the ground that the interpreter would always interpret the texts with his own prejudice. This view was quite different from that in the traditional translation theory which required the interpreter to accurately copy the original text. Obviously, the translator's subjectivity consciousness was awakening under his theory. Gadamer thought it reasonable for the interpreter to have his own prejudice, for it was the premise of understanding and interpretation. Where there is no prejudice, there is no pre- structure for interpretation, thus making it impossible to bring about interpretation. Fundamentally, understanding was the conversational process between the interpreter and the object of interpretation; consequently the textual meaning was generated when the reader and the text achieved "fusion of horizons" during their dialogue process. The translator, as the interpreter with his own unique "horizon", was gradually highlighting his subjectivity identity.

The interpreter aims to explore the author's intention by interpreting the original text, whereas the author attempts to make himself understood through creating the text. In order to unite the two aspects, the two different cultural "horizons" are required to be fused, that is "fusion of horizons". The process of "fusion of horizons" is actually the logical form of the question-answer dialogue between the text and the interpreter. The text casts questions at the interpreter, who in turn employs his own "pre-understanding" to generate his initiatives to actively involve himself in and conduct dialogues with the text. Then the interpreter manages to achieve a "fused" state of the text's "horizon" and the interpreter's "horizon" through the collision and friction of their "horizons". It is just during the constant dialogues between the interpreter and the object to be interpreted that the initiative of the interpreter in the interpretation activities has been fully displayed, making the translator's actively subjective involvement steeping onto a newly high level. Gadamer's notion of "fusion of horizon" implies the essence of the translation, especially the literary translation, namely the translator is supposed to get close to the primitive horizon of the original author, so much so that they could understand the author's original meaning. [5]It is clear that the translator cannot discard his own horizon just like what the traditional translation theories required to fit in with other's horizons. In terms of the literary translation, the translator's subjectivity has become especially prominent under the notion of "fusion of horizon". Therefore translating is not a simple process of copy and the translator, as the first reader, is not a "translation machine" for only simple duplication and imitation, which would be accounted for that the two different "horizons" cannot be in perfect accordance with each other in all-round way. Similarly, the translating process is not a simple shift in the textual languages and the translator' "creative rebellion" against the original text is inevitable, in other words, the translator's subjectivity identity has been recognized and reconstructed.

The process of "fusion of horizon", of course, is not a smooth and gentle one, but one with violent and intense collision, friction and rejection of the two "horizons". During this process, the translator would make his choice and decision on the translation strategies according to his own unique horizon. Put it in other way, it is essential to filter the culture of the original text. The translator is required to actively make a trade-off when filtering, either discarding his unique cultural outlook to approach to the original culture or domesticating the images and implications of the original-text culture into the target-language cultural symbols. Similarly, the process of making the choice is also the adequate evidence of the prominent highlight of the translator's subjectivity identity. 
In the final analysis, from the perspective of historical interpretation, Gadamer suggested that the interpreter had his own "prejudice" and different "prejudices" would offer different "horizons". During the process when the translator's "horizon" and the author's "horizon" were fusing, different translators would surely leave a certain kind of subjective trace on the translated text when doing the translation, thus increasingly highlighting the translator's identity. In Gadamer's philosophy, especially under his notion of "fusion of horizons", the translator jumped out of the framework set for themselves by the traditional translation theories. And he could make his own choices of the translation strategies and methods according to the times' needs and his own horizons. Such practices as mentioned above were quite different from these in the traditional translation theories, under whose direction the translator were required to abandon his subjective infiltration so as to objectively and passively explore the author's original intention as well as the meaning of the text. Consequently, the author's authority had been digested away, whereas the translator had become the main person who was equal to the author and could have dialogues with the latter. Therefore, the translator's identity had been undoubtedly highlighted.

\section{Reflection and Conclusion}

Although the importance of the translator has been gradually revealed with the further study of the translation theories, the cognition of the translator's central position and dominant role has not changed essentially. In addition, the professional image and status of the translator have not been improved substantially. As a matter of fact, we have been used to stating that we are reading the work of some author's original text while reading the translated work of some translator. Thus it is indispensable to obtain a fair and comprehensive cognition of the translator as well as acquire a correct perspective of the translator's position if we want to make a new, complete and systematic depiction and interpretation of the translation process. As time goes by, however, the scholars in the translation field have gradually recognized the fact that the traditional translation theories had not adequately constructed the identity of the translator. In the 1980s, the emergence of the "cultural turn" in the translation theory world opened up a new perspective and a broader horizon for translation studies. Consequently, translation studies have gradually shifted from the ontology research to the subject research. The notion of "fusion of horizons" offered a new prospect, a new concept and a new approach for the reconstruction of the translator's identity. Under this notion, the translator's own personality and initiative have been unprecedentedly highlighted, which has a strong impact on the translator's identity of being a servant in the traditional translation theories, leading the reconstruction of the translator's identity.

\section{References}

[1] Simon, Sherry. Gender in Translation Cultural Identity and the Politics of Transmission. London: Routledge, 1996.

[2] Jouve. N. W. Wllite. Woman Speaks with Forked Tongue: Criticism as Autobiography. London and New Yor: Routledg, 1991.

[3] Ge Xiaoqin. "Shackles of translator's sugjectivity: from source text to target text," Foreign Languages Research, 2002 (1).

[4] J. Weinsheimer, Gadmer's Hermeneutics: A Reading of Truth and Method, 1985.

[5] Xie Tianzhen. "Author's Original Intention, Textual Meaning," Journal of Foreign Languages, 2000 (3). 